\title{
Innominate artery stenting: a single centre experience
}

\begin{abstract}
Objectives: To analyze retrospectively the procedures of PTA with stenting of the innominate artery lesions performed in our Centre.

Background: Endovascular interventions have revolutionized the contemporary treatment of periferal vascular atherosclerotic disease. Innominate artery lesions have traditionally been treated with surgical extra-anatomic bypass via a cervical approach or median sternotomy. Percutaneous transluminal angioplasty (PTA) with stenting may be a viable alternative to traditional open by-pass.

Methods: Between January 2002 and March 2009, 6 patients affected by innominate artery lesions (1 occlusion and 5 stenoses) were treated with PTA and stent using an humeral artery approach. All patients were symptomatic (previous transient ischemic attack, vertebrobasilar insufficiency, upper right limb claudicatio). Short and midterm follow-up included: physical examination, comparative blood pressure measurement on both arms and eco-Doppler examination of the supra-aortic truncks.
\end{abstract}

Results: In all cases we obtained a successful immediate angiographyc result. No major complication (death, stroke) occurred during the immediate perioperative period and troughout all the follow up. Minor complications included puncture site hematoma (1 patients) and acute renal insufficiency (1 patient with known cronic renal insufficiency). Symptoms improved in all patients. Throughout follow up there have been no cases of restenosis detected by routine eco-Doppler examinations and all patients have remained symptoms free.

Conclusion: Percutaneous transluminal angioplasty with stenting may be a viable, effective and safety alternative to traditional surgical treatment of the innominate artery stenosis and occlusions.

Keywords: innominate artery, PTA, TIA, vertebrobasilar insufficiency, upper limb claudication, stent
Volume 6 Issue 4 - 2016

\author{
Moncalvo C, Puma V, Laurenza A, Carosio G, \\ Cioffi P \\ Division of Hemodynamics, Cardiovascular Department, NNC \\ Citt
}

Correspondence: Cinzia Moncalvo, Divisione di Emodinamica, NCC Clinica Città di Alessandria, via V. Moccagatta, 30 - 15121 Alessandria $\square$ Italy, Email czandra@libero.it

Received: August 16, 2016| Published: September 07, 2016

\section{Introduction}

Innominate and common carotid artery lesions have usually been treated surgically with extra-anatomic bypass via a cervical approach or median sternotomy. The transtoracic approach succeeded in providing direct access to proximal aortic arch branch vessel lesions, but these approach was associated with rather high mortality rate $(22 \%) .{ }^{1-4}$ In 1967, Diethrich et al., ${ }^{5}$ reported the first extra-thoracic supraortic trunks (SATs) reconstruction with a dramatic reduction in the mortality rate (from $22 \%$ to $5.6 \%$ ). ${ }^{5}$ A further evolution in the treatment of atherosclerotic lesions of the SATs occurred in the $1980 \mathrm{~s}$ as percutaneous transluminal angioplasty (PTA) techniques were applied to these lesions. Initially these lesions were treated with PTA alone, ${ }^{6-13}$ but subsequent studies have reported an improvement in these initial results when stenting implantation was associated to balloon angioplasty. ${ }^{11,14-17}$ Recent reports, with short and midterm results, suggest that percutaneous treatment of the innominate arteries can be performed with relatively safety and expectations of satisfactory midterm success. ${ }^{6,-12,18,19}$ In our Center we have performed 6 procedures of PTA and stenting for stenotic and occlusive innominate artery lesions. This paper reports the retrospective analysis of these 6 cases.

\section{Methods}

Between January 2002 and March 2009, 6 patients presenting innominate artery lesion ( 1 occlusion and 5 stenoses) underwent PTA and stenting treatment, by a humeral artery approach, at the Haemodinamic Division of our Center (NCC Clinica Città di
Alessandria - Alessandria). This is a retrospective analysis of these cases. All patients, before performing angiography, underwent preoperative physical examination with bilateral arm pressure measurements and had an eco-Doppler of SATs, performed in our Institute. All procedures were performed under local anesthesia. In our experience the access to the SAT lesion was achieved in a retrograde fashion via a humeral right artery puncture. All patients received an intra-arterial bolus dose of heparin (5000 UI) before the intervention. A precise angiographyc analysis of the dimension of the innominate artery (diameter of the vessel and length of the lesion) and of the morphology of the plaque is fundamental before performing the procedure; a correct strategy of approach reduces complications.

In presence of a fibrotic or fibro-calcific plaque with regular surface we implanted traditional stents (Express, 2 cases). In presence of a soft plaque (with high athero-thrombotic content) or total occlusion we preferred stent-graft instead (Jomed, 4 cases), with the intent of positioning the stent-graft immediately after having crossed the lesion with the sheath, in this way most of the plaque and her eventual thrombotic content could be entrapped between the stent and the artery walls reducing the risk of distal atheroembolization in carotid or vertebral artery as distal embolic protection were not employed.

Before implantation of stent graft we crimped manually the stent on a balloon of suitable diameter and length, taking care not to choose a large balloon, which could have difficulty in passing into the sheath, and being careful not to dislocate the stent from its balloon. 
After having chosen the type of stent, its diameter and its length we proceeded to percutaneous puncture of the right humeral artery and we placed a 7 or $8 \mathrm{~F}$ Arrow-Flex sheath, $45 \mathrm{~cm}$ in length with the intent of placing the sheath in the ascending tract of the aorta. In presence of total occlusion of the innominate artery, before crossing the lesion with the sheath, we passed the occluded tract with a coaxial system represented by a $5 \mathrm{~F}$ Vertebral catheter with a 0.035 -in straight Terumo guidewire. We performed this technique to be less traumatic as possible and with the purpose of carrying the stent to the lesion site, keeping it running into the sheath. After having checked the correct locations of the stent, the sheath was withdrawn proximally and the stent was released. The most suitable angiography projection for the release of the stent is the left oblique, the right oblique projection is used to evaluate the bifurcation of the innominate artery into its branches: the right subclavian artery and the right common carotid artery.

We kept the stent stretching out from 2 to $5-\mathrm{mm}$ in the aortic arch to cover the innominate artery ostium. Ostial calcifications were helpful for the right implantation of the stent and they should be entirely covered, also the presence of a Pig-tail, running parallel to the convex portion of the aortic arch, helped us to release the stent correctly, with a slight protrusion in the arch. In order to be sure to cover completely the ostium of the innominate artery with the stent we laid the proximal marker of the balloon of the stent on the Pig-tail catheter. After stent implantation further postdilatation were performed until the optimal diameter was obtained. The innominate artery ostium was usually dilated with high pressure, obtaining a slight flaring. Particular attention should be kept in dilating the distal segment of the stent using angioplasty-balloon with the same diameter of the vessel to avoid a dissection at distal edge of the stent, this complication is very difficult to treat if the dissection involves the ostium of the right common carotid artery. Great care should be given in oversizing the ostium of the innominate artery, by postdilatation, in presence of large calcifications involving the aortic arch to avoid a dissection of the aortic wall, so when these large calcifications are detected we suggest to prefer even a suboptimal expansion of the stent, especially if the previous pressure gradient is cleared.

Physical examinations with bilateral arm pressure measurements were obtained postoperatively and during hospital stay in all patients, an eco-Doppler of SATs was performed after 1months, six months and at 12 months intervals thereafter if clinical conditions were stable. All patients received antiplatelet therapy: ASA (1 case), Ticlopidine ( 2 cases), ASA + Ticlopidine ( 2 cases), ASA + Clopidogrel (1 case).

\section{Result}

Between January 2002 and March 2009, 6 patients (4 males and 2 females) presenting innominate artery lesion (1 occlusion and 5 stenoses) underwent PTA and stenting treatment, via a humeral right artery puncture. Mean age was 59, 3years (range from 49 to 78 years). All patients suffered from hypertension, 2 patients presented dyslipidemia and 1 presented diabetes mellitus. Symptoms were present in all patients and included previous transient ischemic attack (2), vertebrobasilar insufficiency (2), upper right limb claudication (4). The overall mean stenosis treatment rate was $94 \%$ (range $90-100 \%$ ) which was determined by preoperative angiography. One patient had already received PTA alone of the innominate artery 11years before. 2 patients received traditional stents (Express stent), the other patients (4) received a stent-graft (Jomed stent graft).

Mean length of hospital stay was 2days when no complication occurred. In all cases we obtained a successful immediate angiography result. No major complication (death, myocardial infarction, stroke) occurred during the immediate perioperative period and trough out all the follow up. Two minor complications occurred in the postoperative period: one patient, with known chronic renal insufficiency, developed an acute renal failure induced by medium contrast, he was treated with dopamine at renal dosage and hydratation with reduction of creatinine values (at discharge creatinine values were similar to the those of the admission). Another patients presented hematoma at puncture sites (humeral right artery and femoral right artery), treated with manual compressions and compressive dressings without sequela. All patients have remained symptoms free throughout followup and eco-Doppler controls of SATs documented the maintenance of the good angiography result.

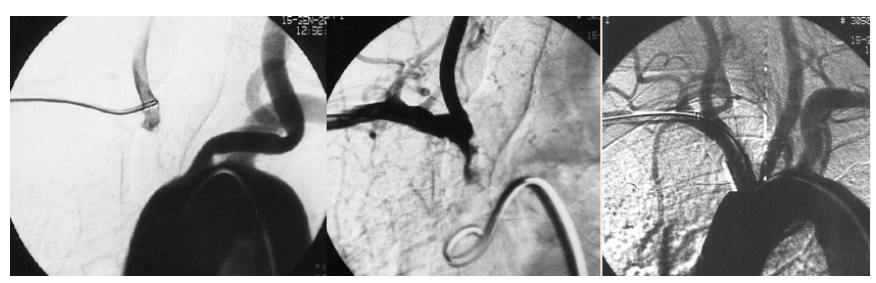

Figure I Successful innominate artery PTA and stenting in a 50-year-old woman.

(A):Angiography showed a total ostial occlusion of the innominate artery.

(B): The innominate artery was dilated with PTA with an angioplasty balloon of $8 \mathrm{~mm}$ of diameter.

(C): Control angiography following stent graft implantation: no residual stenosis can be seen.

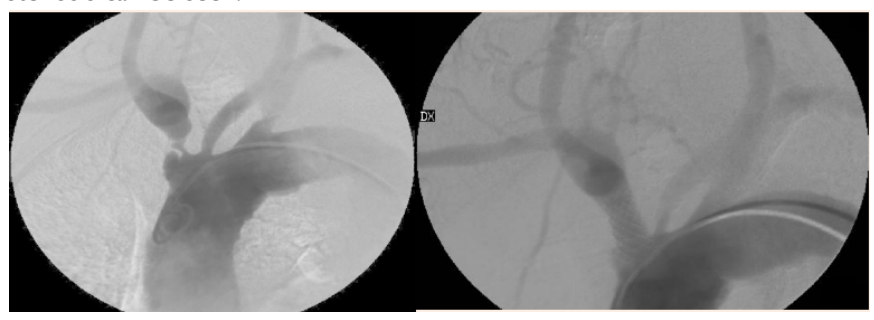

Figure 2 Innominate artery stenting in a 56-year-old man.

(A):Angiography showed a soft and irregular plaque conditioning a subocclusive stenosis of the innominate artery.

(B): Control angiography following direct stent graft implantation.

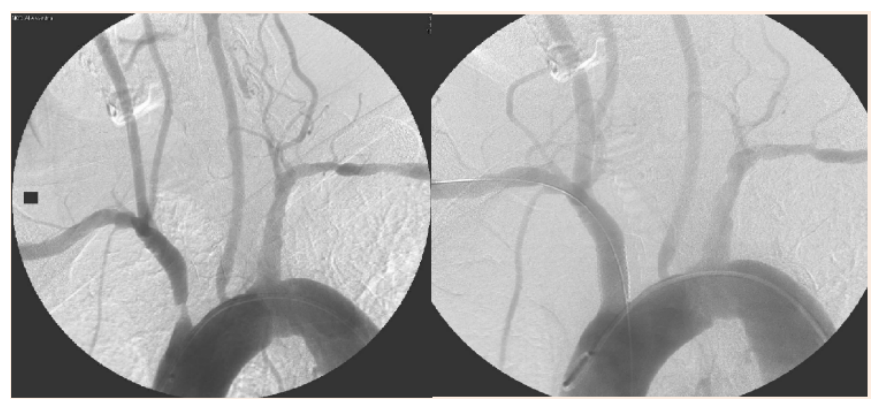

Figure 3 Innominate artery PTA followed by stenting implantation in a 66-year-old man.

(A):Angiography showed a $90 \%$ stenosis of the innominate artery.

(B): Final angiography: the stenosis was dilated with an angioplasty balloon of 5 $\mathrm{mm}$ of diameter, then a traditional stent was implanted.

\section{Discussion}

The treatment of atherosclerotic SAT vessel stenosis has experienced a variety of approaches since 1951 when Shimizu and Sano reported the first surgical repair of those lesions. ${ }^{1}$ The 
transtoracic approach succeeded in providing direct access to proximal aortic arch branch vessel lesions, but these approach was associated with rather high mortality rate $(22 \%))^{1-4}$ In an effort to improve the mortality rate seen with transthoracic repair, in 1967 Diethrich et al., ${ }^{5}$ reported the first extra-anatomic SAT reconstruction; that improvement was subsequently confirmed by Crawford et al., ${ }^{4}$ who demonstrated a reduction in the mortality rate from $22 \%$ to $5.6 \%$ with extra-anatomic cervical repair. Following reports of the successful treatment of peripheral, renal and coronary artery atherosclerotic lesions by PTA, ${ }^{20,21}$ in the 1980 s PTA techniques were applied to the lesions of SATs. ${ }^{9,10}$ While these interventions were well tolerated by most patients and demonstrated acceptable perioperative efficacy and safety, long term patency and freedom from reintervention were found to be inferior to the surgical treatment. Stenting was subsequently introduced to address these issues and theoretically reduce the risk of atheroembolization. Recent experiences of PTA and stenting treatment of the innominate artery lesions, performed in other center, with short and midterm results, suggest that percutaneous treatment of the innominate arteries can be performed with relatively safety and expectations of satisfactory mid-term success. ${ }^{6,-12,18,19}$ In our experience the access to innominate artery lesion was achieved in a retrograde fashion via a humeral right artery puncture. The antegrade access via a femoral artery puncture, in our opinion, is rather uncomfortable because it implies great difficulties in crossing the steno-occlusive lesions with the guidewires, it doesn't supply enough stability of the catheters and of the sheaths carrying the stent, it doesn't allow the correct location of the stent which should stretch out in the aortic arch and it doesn't allow the release of other stents if a distal dissection occurs; all these problems can be achieved in presence of a winding aortic arch (type III aortic arch or bovine aortic arch) and in presence of great calcifications or atherotrombosis of the aortic arch with a high risk of cerebral embolization, while sounding the vessel.

The procedure is more comfortable and safe using a retrograde humeral approach and complications are really reduced. In our procedures we always preferred a humeral access, one of the possible complications of the humeral puncture is hematoma at puncture site due to a difficult manual compression. In our experience we have never used the right radial approach, even if it would avoid hematomas and manual compression would be easier, because for these procedures we employed 7 and $8 \mathrm{~F}$ sheaths. A precise angiographyc analysis of the dimension of the innominate artery (diameter of the vessel and length of the lesion) and of the morphology of the plaque is fundamental before performing the procedure; a correct strategy of approach reduces complications. Computed tomography angiograms are useful tools for the evaluation of the morphology of the plaque; the different type of plaque and its associate risk of embolization make us decide for a different kind of stent (stent graft or traditional stent). Particular attention should be kept in dilating the distal segment of the stent at these purpose we used angioplastyc-balloon with the same diameter of the vessel to avoid a dissection at distal edge of the stent, this complication is very difficult to treat most of all if the dissection involves the ostium of the right common carotid artery. Great care should be given in oversizing the ostium of the innominate artery, during postdilatation, in presence of large calcifications involving the aortic arch to avoid a dissection of the aortic wall, so when these large calcifications are detected we suggest to prefer a suboptimal expansion of the stent, especially if the previous pressure gradient is cleared.

All the six patients treated in our Center with this technique showed immediately a good angiographic result, symptoms disappeared and throughout the follow up there have been no cases of restenosis, no late target vessel-related complications or deaths.

\section{Conclusion}

The percutaneous approach has many theoretical advantages over open surgical repair including the ability to perform such procedures without the need for general anesthesia, quicker recovery times, shorter length of hospital stay and lower costs (Takach et al., ${ }^{22}$ reported a mean saving of $\$ 8787$ per procedure. ${ }^{22}$ ) High-risk patients such as those suffering cardiac events and others with significant comorbidities, previous cervical operations, or prior cervical irradiation are likely to benefit from an endovascular approach to innominate artery stenosis or occlusions.

In suitable patients percutaneous transluminal angioplasty with stenting may be a viable, effective and safety alternative to traditional surgical treatment of the innominate artery stenosis and occlusions. Particular attention should be kept in case of total occlusion of the innominate artery because of the risk of cerebral embolization.

\section{Acknowledgments}

None.

\section{Conflicts of interest}

Author declares there are no conflicts of interest.

\section{Funding}

None.

\section{References}

1. Shimizu K, Sano K. Pulseless disease. J Neuropathol Clin Neurol. 1951;1(1):37-47.

2. Davis JB, Grove WJ, Julian OC. Thrombic occlusion of branches of aortic arch, Martorell's syndrome: report of case treated surgically. Ann Surg. 1956;144(1):124-126.

3. DeBakey ME, Morris GC, Jordan GL, et al. Segmental thromboobliterative disease of branches of the aortic arch. J Am Med Assoc. 1958;166(9):998-1003.

4. Crawford ES, DeBakey ME, Morris GC, et al. Surgical treatment of occlusion of the innominate, common carotid, and subclavian arteries: a 10-year experience. Surgery. 1969;65(1):17-31.

5. Diethrich EB, Garrett HE, Ameriso J, et al. Occlusive disease of the common carotid and subclavian arteries treated by carotidsubclavian bypass: analysis of 125 cases. Am J Surg. 1967;114(5):800-808.

6. Motarjeme A. Percutaneous transluminal angioplasty of supra-aortic vessels. J Endovasc Surg. 1996;3(2):171-181.

7. Henry M, Amor M, Henry I, et al. Percutaneous transluminal angioplasty of the subclavian arteries. J Endovasc Surg. 1999;6(1):33-41.

8. Huttl K, Nemes B, Simonffy A, et al. Angioplasty of the innominate artery in 89 patients: experience over 19 years. Cardiovasc Intervent Radiol . 2002;25(2):109-114.

9. Dorros G, Lewin RF, Jamnadas P, et al. Peripheral transluminal angioplasty of the subclavian and innominate arteries utilizing the brachial approach: acute outcome and follow-up. Cathet Cardiovasc Diagn. 1990;19(2):71-76.

10. Insall RL, Lambert D, Chamberlain J, et al. Percutaneous transluminal angioplasty of the innominate, subclavian, and axillary arteries. Eur $J$ Vasc Surg . 1990;4(6):591-595.

11. Sullivan TM, Gray BH, Bacharach JM, et al. Angioplasty and primary stenting of the subclavian, innominate, and common carotid arteries in 83 patients. J Vasc Surg. 1998;28(6):1059-1065. 
12. Vitek JJ, Raymon BC, Oh SJ. Innominate artery angioplasty. Am J Neuroradiol . 1984;5(1):113-114.

13. De Vries JP, Jager LC, Van den Berg JC, et al. Durability of percutaneous transluminal angioplasty for obstructive lesions of proximal subclavian artery: longterm results. J Vasc Surg. 2005;41(1):19-23.

14. Brountzos EN, Petersen B, Binkert C, et al. Primary stenting of subclavian and innominate artery occlusive disease: a single center's experience. Cardiovasc Intervent Radiol. 2004;27(6):616-623.

15. Hadjipetrou P, Cox S, Piemonte T,et al. Percutaneous revascularization of atherosclerotic obstruction of aortic arch vessels. J Am Coll Cardiol . 1999;33(5):1238-1245.

16. Whitbread T, Cleveland TJ, Beard JD, et al. A combined approach to the treatment of proximal arterial occlusions of the upper limb with endovascular stents. Eur J Vasc Endovasc Surg. 1998;15(1):29-35.

17. Rodriguez-Lopez JA, Werner A, Martinez R, et al. Stenting for atherosclerotic occlusive disease of the subclavian artery. Ann Vasc Surg. 1999;13(3):254-260.
18. Queral LA, Criado FJ. The treatment of focal aortic branch lesions with Palmaz stents. J Vasc Surg . 1996;23(2):368-375.

19. Kálmán H, Balázs N, Árpád S, et al. Angioplasty of the Innominate Artery in 89 Patients: Experience over 19 Years. Cardiovasc Intervent Radiol . 2002;25(2):109-114.

20. Grüntzig AR, Senning A, Siegenthaler WE. Nonoperative dilatation of coronaryartery stenosis: percutaneous coronary angioplasty. $N$ Engl $J$ Med. 1979;301(2):61-68.

21. Zeitler E, Richter EI, Roth FJ, et al. Results of percutaneous transluminal angioplasty. Radiology. 1983;146(1):57-60.

22. Takach TJ, Duncan JM, Livesay JJ, et al. Brachiocephalic reconstruction II: operative and endovascular management of single-vessel disease. $J$ Vasc Surg . 2005;42(1):55-61. 